\title{
DETECTION OF COUPLING ELEMENTS IN CESR*
}

\author{
D. Sagan \\ Wilson Laboratory, Cornell University, Ithaca NY, 14853, USA
}

\section{Abstract}

At the Cornell Electron/positron Storage Ring CESR, the measurement of the coupling between the horizontal and vertical beam motions has led to the ability to locate the position of a coupler and to calculate its strength. Once the identity of a coupler is known, steps can be taken to remove it or to nullify its effect. This coupling analysis can also be used to calibrate correction skew quadrupoles.

\section{INTRODUCTION}

In a storage ring, the presence of couplers, which couple the horizontal and vertical motions of a beam, can lead to an increase in the vertical beam size with an attendant loss in luminosity[1]. In order to be able to keep the coupling under control, a technique for measuring the coupling has been developed at the Cornell Electron/positron Storage Ring CESR[2]. This involves shaking the beam at the betatron resonant frequencies and measuring the response at the 100 or so detectors in the ring. In order to be able to find unwanted sources of coupling, an analysis program has been developed that can locate isolated coupling elements. This analysis is presented below[3] and is analogous to the technique of using orbit data to find isolated steering kicks. As an added benefit, the analysis can also be used to calibrate the strength of skew quadrupoles.

\section{ANALYSIS}

\subsection{How the Coupling is Defined}

Any longitudinal oscillations are ignored and, following Sagan and Rubin[4], the $4 \times 41$-turn transfer matrix $\mathbf{T}(s)$ is written in normal mode form

$$
\mathbf{T}=\mathbf{V} \mathbf{U} \mathbf{V}^{-1},
$$

where the normal mode matrix $\mathbf{U}$ is of the form

$$
\mathbf{U}=\left(\begin{array}{cc}
\mathbf{A} & \mathbf{0} \\
\mathbf{0} & \mathbf{B}
\end{array}\right)
$$

and $\mathbf{V}$ is of the form

$$
\mathbf{V}=\left(\begin{array}{cc}
\gamma \mathbf{I} & \mathbf{C} \\
-\mathbf{C}^{+} & \gamma \mathbf{I}
\end{array}\right)
$$

with "+" denoting the symplectic conjugate. Since $\mathbf{V}$ is required to be symplectic, $\gamma$ and $\mathbf{C}$ are related by

$$
\gamma^{2}+\|\mathbf{C}\|=1 .
$$

\footnotetext{
${ }^{*}$ Work supported by the National Science Foundation
}

$\mathbf{C}(s)$ is a measure of the coupling; if $\mathbf{C}=\mathbf{0}$ then $\mathbf{V}=\mathbf{1}$ and $\mathbf{T}$ is decoupled. Instead of working with $\mathbf{C}$, though, it is convenient to work with the normalized matrix $\overline{\mathbf{C}}$ given by

$$
\overline{\mathbf{C}}=\mathbf{G}_{a} \mathbf{C ~ G}_{b}^{-1},
$$

where $\mathbf{G}_{a}$ and $\mathbf{G}_{b}$ are normalization matrices for the $a$ and $b$ normal modes respectively given by

$$
\mathbf{G}=\left(\begin{array}{cc}
\frac{1}{\sqrt{\beta}} & 0 \\
\frac{\alpha}{\sqrt{\beta}} & \sqrt{\beta}
\end{array}\right) .
$$

At CESR, $\overline{\mathbf{C}}$ is measured by observing the horizontal and vertical oscillation of the beam at the detectors in the ring while shaking the beam at a betatron resonance[2]. In order to be able to extract the strength and location of coupling elements from the data, it is necessary to know how $\overline{\mathbf{C}}$ changes across a coupler, and how $\overline{\mathbf{C}}$ propagates in a region where there are no coupling elements.

\section{$2.2 \overline{\mathbf{C}}$ Variation}

If there are no couplers in a local region then the variation of $\overline{\mathbf{C}}$ is given by[4]

$$
\overline{\mathbf{C}}(s)=\lambda \mathbf{S}\left(\phi_{0}-\phi_{+}(s)\right)+\kappa \mathbf{R}\left(\theta_{0}+\phi_{-}(s)\right),
$$

where $\lambda, \phi_{0}, \kappa$, and $\theta_{0}$ are constants, $\mathbf{R}$ and $\mathbf{S}$ are rotation and "anti-rotation" matrices of the form

$$
\mathbf{R}(\theta) \equiv\left(\begin{array}{cc}
\cos \theta & \sin \theta \\
-\sin \theta & \cos \theta
\end{array}\right),
$$

and

$$
\mathbf{S}(\phi) \equiv\left(\begin{array}{cc}
\cos \phi & \sin \phi \\
\sin \phi & -\cos \phi
\end{array}\right),
$$

with the sum and difference phase advances being

$$
\begin{aligned}
& \phi_{+} \equiv \phi_{a}+\phi_{b}, \quad \text { and } \\
& \phi_{-} \equiv \phi_{a}-\phi_{b} .
\end{aligned}
$$

To compute the change in $\overline{\mathbf{C}}$ across a coupler, it is assumed that the coupling is small so that terms second order in $\mathbf{C}$ (or $\overline{\mathbf{C}}$ ) may be neglected. Thus, from Eq. (4), to first order in the coupling

$$
\gamma=1
$$

Using this, and Eqs. (1), (2), and (3), gives to first order

$$
\mathbf{T}=\left(\begin{array}{cc}
\mathbf{A} & \mathbf{C B}-\mathbf{A C} \\
\mathbf{B C}^{+}-\mathbf{C}^{+} \mathbf{A} & \mathbf{B}
\end{array}\right) .
$$

Eq. (12) shows that the on-diagonal $2 \times 2$ submatrices of $\mathbf{T}$ are unaffected by the coupling. Thus, to first order, the 
eigenmode betas, $\beta_{a}$ and $\beta_{b}$ and the eigenmode phases, $\phi_{a}$ and $\phi_{b}$, are equal to what would be calculated if the coupling is absent.

Couplers are modeled as thin skew quadrupoles. The $4 \times 4$ transfer matrix $\mathbf{T}_{\text {coup }}$ for a coupler is then

$$
\mathbf{T}_{\text {coup }}=\left(\begin{array}{cc}
\mathbf{1} & -\mathbf{q} \\
-\mathbf{q} & \mathbf{1}
\end{array}\right)
$$

where

$$
\mathbf{q}=\left(\begin{array}{cc}
0 & 0 \\
\delta q & 0
\end{array}\right)
$$

with $\delta q$ being the strength of the coupler. The 1-turn matrix $\mathbf{T}_{0+}$, located at a point just after a coupler, is related to the 1-turn matrix $\mathbf{T}_{0-}$, located at a point just before the coupler, via

$$
\mathbf{T}_{0+}=\mathbf{T}_{\text {coup }} \mathbf{T}_{0-} \mathbf{T}_{\text {coup }}^{-1}
$$

Multiplying out Eq. (15), and comparing with Eq. (12), gives, to first order, and with the help of Eq. (5)

$$
\overline{\mathbf{C}}_{0+}=\overline{\mathbf{C}}_{0-}-\overline{\mathbf{q}}
$$

where

$$
\overline{\mathbf{q}}=\left(\begin{array}{cc}
0 & 0 \\
\delta \bar{q} & 0
\end{array}\right)
$$

with

$$
\delta \bar{q}=\sqrt{\beta_{a} \beta_{b}} \delta q
$$

\subsection{Isolated Coupler Analysis}

Given a coupler of interest at some point $s_{0}$, it is assumed that the coupler is "isolated" so that it is the only coupler in some local region. Since we have linearized the problem, the general solution for $\overline{\mathbf{C}}$ is the sum of a homogeneous part $\overline{\mathbf{C}}_{h}$ plus an inhomogeneous part $\overline{\mathbf{C}}_{i}$

$$
\overline{\mathbf{C}}(s)=\overline{\mathbf{C}}_{h}(s)+\overline{\mathbf{C}}_{i}(s) .
$$

The homogeneous part is the solution when there is no coupler and is given by Eq. (7). The inhomogeneous part is the solution with the coupler and with some boundary condition which we are free to choose. This boundary condition will be chosen to be $\overline{\mathbf{C}}(s)=\mathbf{0}$ for $s<s_{0}$. From Eqs. (7) and (16), the inhomogeneous solution is

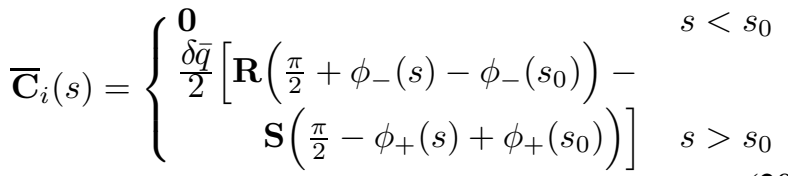

At CESR, the coupling measurement can measure the $\bar{C}_{11}, \bar{C}_{12}$, and $\bar{C}_{22}$ components of $\overline{\mathbf{C}}$. For various technical reasons, the errors in the $\bar{C}_{12}$ data are less than the errors present in the measurement of the other components. Therefore, the following analysis will consider only the
$\bar{C}_{12}$ component. Extending the analysis to the other components is a trivial matter. From Eq. (7), the general solution for $\bar{C}_{12}$ can be written as

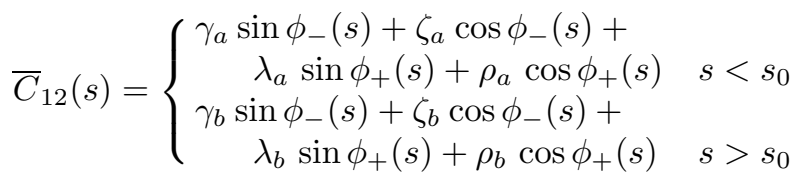

The procedure for locating a coupler is as follows: Given a putative coupler location, two regions are chosen. One region, labeled "A", is chosen to be just before the coupler. The other region, labeled " $\mathrm{B}$ ", is chosen to be just after the coupler (See the example at the end of the paper). Since the betatron phase is, to first order, independent of the coupling, the phase is taken to be equal to the phase in the design lattice. With this, the sine and cosine functions in Eq. (21) may be evaluated and then a least squares fit can be used to determine $\gamma_{a}, \zeta_{a}, \lambda_{a}$, and $\rho_{a}$ using the data from the A region. Similarly, $\gamma_{b}, \zeta_{b}, \lambda_{b}$, and $\rho_{b}$ are obtained from a least squares fit using the data from the $\mathrm{B}$ region. The inhomogeneous part of the solution is now obtained from Eq. (21) by subtracting out the homogeneous part to give

$$
\bar{C}_{i, 12}= \begin{cases}0 & s<s_{0} \\ \gamma_{b a} \sin \phi_{-}(s)+\zeta_{b a} \cos \phi_{-}(s)+ & \\ \lambda_{b a} \sin \phi_{+}(s)+\rho_{b a} \cos \phi_{+}(s) & s>s_{0}\end{cases}
$$

with

$$
\begin{aligned}
\gamma_{b a} & =\gamma_{b}-\gamma_{a}, \quad \zeta_{b a}=\zeta_{b}-\zeta_{a}, \\
\lambda_{b a} & =\lambda_{b}-\lambda_{a}, \quad \rho_{b a}=\rho_{b}-\rho_{a} .
\end{aligned}
$$

The phase at the coupler is found by comparing Eq. (20) with Eq. (22)

$$
\begin{aligned}
\tan \phi_{+}\left(s_{0}\right) & =\frac{\lambda_{b a}}{\rho_{b a}}, \quad \text { and } \\
\tan \phi_{-}\left(s_{0}\right) & =\frac{\gamma_{b a}}{\zeta_{b a}} .
\end{aligned}
$$

There are multiple solutions to Eqs. (24) spaced $\pi$ apart. However, valid solutions must have the corresponding $\phi_{a}$ and $\phi_{b}$ (from Eq. (10)) corresponding to a location somewhere between the $\mathrm{A}$ and $\mathrm{B}$ regions.

The magnitude of the coupler is given by comparing Eq. (20) to (22)

$$
\begin{aligned}
& \frac{\delta \bar{q}}{2}=-\lambda_{b a} \sin \phi_{+}\left(s_{0}\right)-\rho_{b a} \cos \phi_{+}\left(s_{0}\right), \quad \text { or } \\
& \frac{\delta \bar{q}}{2}=\gamma_{b a} \sin \phi_{-}\left(s_{0}\right)+\zeta_{b a} \cos \phi_{-}\left(s_{0}\right) .
\end{aligned}
$$

Changes in amplitudes for the $\mathbf{R}$ and $\mathbf{S}$ components of $\bar{C}_{12}$ may be defined by

$$
A_{s, b a}^{2} \equiv \lambda_{b a}^{2}+\rho_{b a}^{2}, \quad A_{r, b a}^{2} \equiv \gamma_{b a}^{2}+\zeta_{b a}^{2} .
$$

Using this, Eqs. (25) may be put in a more transparent form

$$
|\delta \bar{q}|=2 A_{s, b a}=2 A_{r, b a} .
$$

The disadvantage of Eqs. (27), as opposed to Eqs. (25), is that the sign of $\delta \bar{q}$ is lost. 


\subsection{Error analysis}

Even with a good fit to the data, the analysis can be off if there is more than one coupler between the A and B regions. From Eqs. (27), if there is only one coupler, then $A_{s, b a}=A_{r, b a}$. A figure of merit $\chi_{B A}$ can thus be defined by

$$
\chi_{B A} \equiv \frac{\left|A_{s, b a}-A_{r, b a}\right|}{A_{s, b a}+A_{r, b a}} .
$$

The condition for a good fit with only one coupler is then

$$
\chi_{B A} \ll 1
$$

\section{EXAMPLE}

The coupling analysis presented above can be used for finding unwanted sources of coupling, and for calibrating skew quadrupole magnets. Figure 1 shows how coupling data is analyzed. In CESR, there are approximately 100 detectors numbered from 0 to 99 . The $\bar{C}_{12}$ data shown in figure $1 \mathrm{a}$ is the difference between two coupling measurements obtained while varying a skew quadrupole placed between detectors 48 and 49 . The A region was chosen to be between detectors 20 and 48 and the $\mathrm{B}$ region was chosen to be between detectors 50 and 80 . Figure $1 \mathrm{~b}$ shows the $\bar{C}_{12}$ data with the fit to the A region subtracted off. There is a good fit to the data as evidenced by the fact that the plot is near zero within the A region. The general location of the coupler can be located by eye by noting where, just to the right of the A region, the data becomes significantly nonzero. Figure 1c shows the data with the B region fit subtracted off. Again, there is a good fit to the data. Table 1 shows the values of the fitted parameters. From these values, and Eqs. (10), (24) and (25), it is found that there is a single valid solution given by $\phi_{a}=31.86, \phi_{b}=29.70$, and $\delta \bar{q}=0.14$ with $\chi_{B A}=0.09$. This verifies the location of the coupler and gives the magnitude of the kick.

In the above example, the $\mathrm{A}$ and $\mathrm{B}$ regions could be chosen a priori since the location of the coupler was known before hand. When the location is not initially known, the regions can be chosen through trial and error just by looking at the plotted results until a good fit is obtained. If the space between the A and B regions is too wide, Eqs. (24) will have multiple solutions. However, since the solutions are spaced apart in phase (and hence are some distance apart), it is normally a simple matter to be able to select the correct solution.

\begin{tabular}{|c|r|r|r|r|}
\hline Region & $\lambda$ & $\rho$ & $\gamma$ & $\zeta$ \\
\hline$A$ & -0.008 & 0.114 & -0.195 & -0.186 \\
\hline$B$ & 0.057 & 0.093 & -0.128 & -0.231 \\
\hline$B-A$ & 0.065 & -0.021 & 0.067 & -0.045 \\
\hline
\end{tabular}

Table 1: Fitted parameters $\lambda, \rho, \gamma$, and $\zeta$.
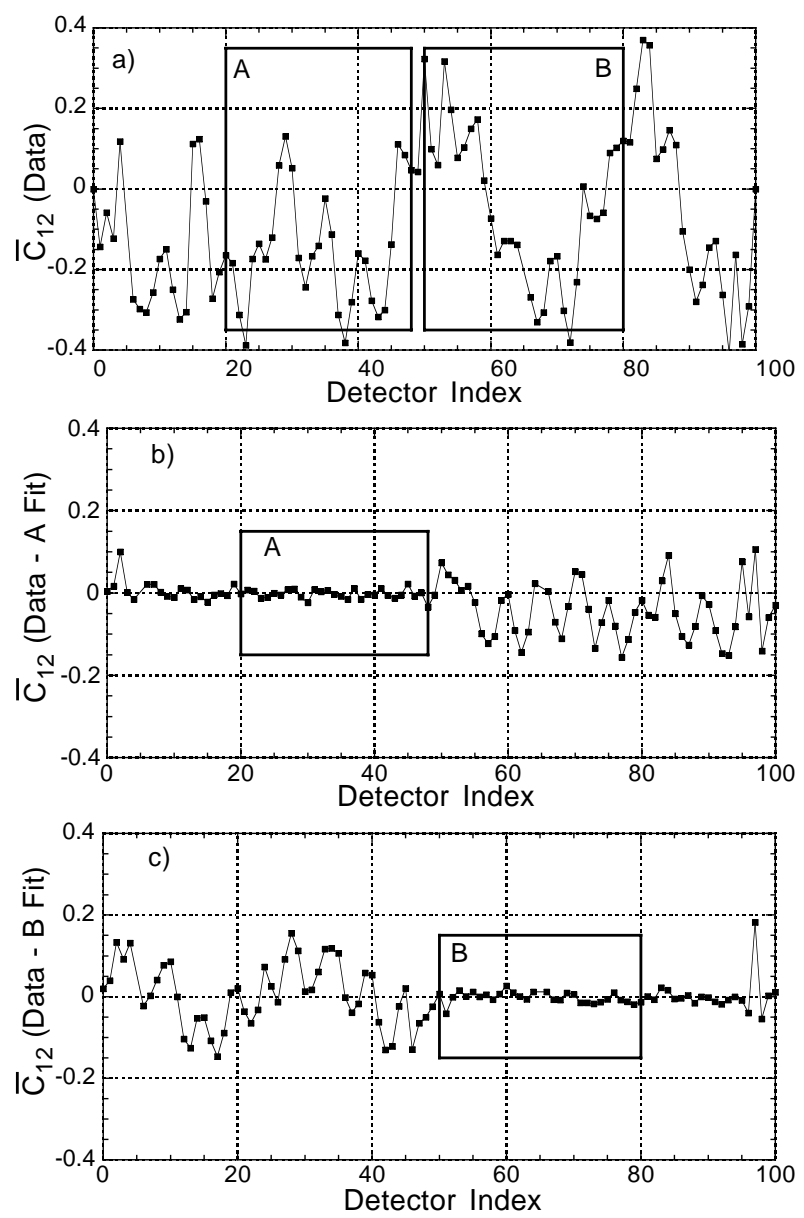

Figure 1: Coupling data. The A and B fit regions are indicated by the boxes. a) Data obtained by varying a skew quadrupole. b) The data with the A region fit subtracted off. c) The data with the B region fit subtracted off.

\section{ACKNOWLEDGEMENTS}

My thanks to Dave Rubin and for useful discussions and to the rest of the CESR operations group for their support.

\section{REFERENCES}

[1] D. Sagan, "The Effect of Coupling on Luminosity Performance," Proc. 1995 Part. Acc. Conf. (San Francisco) p. 3382, (1995).

[2] P. Bagley and D. Rubin, "Correction of Transverse Coupling in a Storage Ring," Proc. 1989 Part. Acc. Conf. (San Francisco) p. 874, (1989).

[3] An expanded version of this paper will be published in the PAC99 special Edition of the on-line journal Phys. Rev. Special Topics - Acc. and Beams (PRST-AB).

[4] D. Sagan and D. Rubin, "Propagation of Twiss and Coupling Parameters," Cornell CBN 96-20 (1996). Can be obtained from the web at: http://w4.Ins.cornell.edu/public/CBN/ 